\title{
Estrategias CRM en empresas de servicios: recomendaciones directivas para su implementación
}

\section{CRM strategies in services companies: managerial recommendations for its implementation}

\author{
Aurora Garrido Moreno y Antonio Padilla Meléndez \\ Facultad de Estudios Sociales y del Trabajo. Universidad de Málaga. Campus de Teatinos. Málaga \\ agarridom@uma.es,apm@uma.es
}

Fecha de recepción: 10-11-2010

Fecha de aceptación: 6-7-2011

Resumen: La Gestión de Relaciones con Clientes (CRM) juega un papel clave en empresas de servicios, en las cuales el contacto con clientes es intensivo, y su fidelización constituye una fuente de ventaja competitiva. Sin embargo, la literatura muestra que no se están obteniendo los resultados esperados con su implementación. En este trabajo presentamos una revisión de la literatura sobre el concepto de CRM y un estudio empírico en 31 I empresas españolas, del que se deriva la propuesta de un marco conceptual para su exitosa implementación en el sector servicios. Como principal recomendación directiva destaca que para tener éxito en su puesta en marcha, debe prestarse más atención a factores de tipo organizativo (formación, estructura organizativa, liderazgo), que a factores tecnológicos.

Palabras claves: estrategias CRM, empresas de servicios, recomendaciones de implementación.

Abstract: Customer Relationship Management (CRM) has a special relevance for service companies, in which contact with customers is intense, and loyalty is a source of competitive advantage. Literature shows that companies are not having the positive expected results in the implementation. In this paper, we summarize a literature review about CRM and an empirical study based on 3 II Spanish companies data. Building upon this, and a structural equation model, we propose a map of relationships of the main factors for a successful CRM implementation. As main managerial implication, we stress that to be successful in implementing CRM, companies should pay more attention to organizational factors than to technological ones.

Key words: CRM strategies, services companies, implementation recommendations.

\section{Introducción}

En el entorno económico actual, en el que las relaciones con el mercado cobran cada vez más importancia, las empresas están centrando su atención en el desarrollo de relaciones personalizadas con los clientes, como medio para alcanzar ventajas competitivas. Particularmente, las empresas de servicios, en las que existe un contacto intensivo con el cliente durante todo el proceso de servucción («fabricación» del servicio), se encuentran especialmente posicionadas para establecer relaciones a largo plazo con clientes (Grönroos, 1994). Así, surge la Gestión de Relaciones con Clientes (Customer Relationship Management, CRM), que consideramos como estrategia de negocio posibilitada por las Tecnologías de la Información (TI), y que implica el establecimiento y desarrollo de relaciones de valor con clientes, dirigidas hacia la retención y lealtad de los mismos. La in- vestigación sobre la temática destaca que a las empresas les resulta más rentable retener a sus clientes existentes, mediante el desarrollo de relaciones a largo plazo que satisfagan sus necesidades, que captar nuevos clientes (Reichheld, 1996).

La presión competitiva, está llevando a las organizaciones a agilizar su cadena de valor completa, transformando estructuralmente sus procesos de negocio, tanto internos como externos, utilizando iniciativas tecnológicas, entre las que destaca el CRM (IglesiasPradas et al., 2009). A pesar de la relativa novedad del concepto de CRM, que surgió en los años noventa, en menos de una década se ha convertido en una herramienta clave para la gestión de empresas actual, y un campo al que la investigación está dedicando una atención preferente (Ngai, 2005; Kevork y Vrechopoulos, 2009; Das, 2009). Dicho interés también se da en las empresas $y$, según datos de la consultora 
Gartner (20|0), el mercado de software de CRM a nivel mundial alcanzará en 2012 los mil millones de dólares, y se espera que siga creciendo a una tasa anual sostenida en los próximos años.

No obstante, a pesar de su relevancia económica y de las numerosas ventajas que, según la literatura, conlleva la implementación del CRM, diversos estudios empíricos muestran también altos índices de fracaso en su puesta en marcha, no obteniéndose, en muchos casos, los resultados esperados a nivel económico y gerencial (Rigby et al., 2002; Xu y Walton, 2005). En este sentido, aunque el concepto de CRM ha sido ampliamente analizado en períodos recientes, siguen sin existir estudios que analicen de manera integral como la implementación de tecnologías CRM se traduce en resultados de negocio, y cuáles son los mecanismos o variables que median esta relación, desde un enfoque integrador (Becker et al., 2009; Reimann et al., 20 I 0; Chang et al., 20 I 0). Son necesarios, por tanto, estudios que se centren en los factores que hacen que el CRM se implemente con éxito y aporte evidencia empírica sobre las relaciones entre dichos factores.

Para cubrir esta necesidad de investigación, en este trabajo presentamos una revisión de la literatura sobre el concepto de CRM y los factores de éxito en su implementación, y un estudio empírico en empresas del sector servicios. Ello nos permite proponer un mapa de relaciones entre los factores que explican cómo realizar una implementación exitosa del CRM (en términos de mercado y financiero), en empresas de servicios.

\section{Concepto de CRM}

El marketing relacional consiste en atraer, mantener e intensificar las relaciones con los clientes (Berry, 1983, p. 25) y es considerado como antecedente y origen del concepto de CRM. Así, diversos autores consideran el CRM como marketing relacional convertido en realidad mediante el uso de TI, con un especial énfasis en la relación con los clientes (Ryals y Paine, 2001 ; Zablah et al., 2004; Reinares y Ponzoa, 2002).

Sin embargo, no existe una descripción o definición generalmente aceptada de lo que supone el CRM. En este trabajo, tras revisar la literatura, hemos agrupado las diversas definiciones (véase Tabla I) en función de su carácter en tecnológicas (CRM como conjunto de tecnologías), estratégicas (CRM como estrategia de negocio) o integradoras (CRM como iniciativa estratégica posibilitada por las TI).

Tabla I

Definiciones de CRM

\begin{tabular}{|l|l|l|}
\hline \multicolumn{1}{|c|}{ Autor } & \multicolumn{1}{|c|}{ Definición } \\
\hline $\begin{array}{l}\text { I. Carácter } \\
\text { tecnológico }\end{array}$ & Choy, Fan y Lo (2003, p.263) & $\begin{array}{l}\text { Supone una integración en toda la empresa de tecnologías traba- } \\
\text { jando conjuntamente como son almacenamiento de datos, sitio } \\
\text { web, intranet-extranet, sistema de apoyo telefónico, contabilidad, } \\
\text { marketing, ventas y producción, para permitir la comunicación en- } \\
\text { tre las distintas partes de la organización y así servir mejor a la } \\
\text { clientela. }\end{array}$ \\
\hline $\begin{array}{l}\text { 2. Carácter } \\
\text { estratégico }\end{array}$ & Parvatiyary Sheth (200I, p. 5) & $\begin{array}{l}\text { El CRM engloba tanto la estrategia como los procesos que com- } \\
\text { prenden la adquisición, retención y asociación con determinados } \\
\text { clientes con objeto de crear un valor superior tanto para la com- } \\
\text { pañía como para el propio cliente. }\end{array}$ \\
\cline { 2 - 4 } & Sigala (2005, p. 393) & $\begin{array}{l}\text { Conjunto de estrategias que tienen la intención de buscar, reco- } \\
\text { pilary almacenar la información adecuada, validarla y compartir- } \\
\text { la a través de toda la organización, con objeto de que después } \\
\text { sea utilizada por todos los niveles organizativos para crear expe- } \\
\text { riencias únicas y personalizadas a sus clientes. }\end{array}$ \\
\hline 3. Enfoque \\
integrador & $\begin{array}{l}\text { Asociación Española de Marketing Re- } \\
\text { lacional (AEMR, 2002, p. 9) }\end{array}$ & $\begin{array}{l}\text { Conjunto de estrategias de negocio, marketing, comunicación e } \\
\text { infraestructuras tecnológicas, diseñadas con el objeto de cons- } \\
\text { truir una relación duradera con los clientes, identificando, com- } \\
\text { prendiendo y satisfaciendo sus necesidades }\end{array}$ \\
\hline
\end{tabular}

(continúa) 
Tabla I

Definiciones de CRM (continuación)

\begin{tabular}{|l|l|l|}
\hline \multicolumn{1}{|c|}{ Autor } & \multicolumn{1}{c|}{ Definición } \\
\hline & Chen y Popovich (2003, p. 673) & $\begin{array}{l}\text { El CRM no es sólo una aplicación tecnológica, es una estrategia } \\
\text { de negocio que aglutina las funciones de marketing, ventas, ser- } \\
\text { vicio al cliente, operaciones, recursos humanos, I+D, finanzas y } \\
\text { Tl con el objeto de maximizar la rentabilidad de las interaccio- } \\
\text { nes con clientes. }\end{array}$ \\
\hline $\begin{array}{l}\text { 3. Enfoque } \\
\text { integrador }\end{array}$ & Renart (2004, p. 6) & $\begin{array}{l}\text { El CRM hace referencia tanto a la estrategia de negocio enfoca- } \\
\text { da a seleccionary gestionar una relación con los mejores clientes } \\
\text { para optimizar su valor a largo plazo, como a las aplicaciones con- } \\
\text { cretas de software necesarias para procesar la información de } \\
\text { esos clientes y desarrollar esa relación. }\end{array}$ \\
\hline
\end{tabular}

Fuente: Elaboración propia.

Como resultado de dicho análisis, definimos el CRM a modo de síntesis, como estrategia de negocio que persigue el establecimiento y desarrollo de relaciones de valor con clientes, basadas en el conocimiento. Utilizando las TI como soporte, el CRM implica un rediseño de la organización y sus procesos para orientarlos al cliente, de forma que, por medio de la personalización de su oferta, la empresa pueda satisfacer óptimamente las necesidades de los mismos, generándose relaciones de lealtad a largo plazo, mutuamente beneficiosas.

\section{Implementación del CRM: errores y factores de éxito}

La implementación del CRM conlleva numerosas ventajas para la empresa (Peppard, 2000; Chen y Popovich, 2003), que se concretan en un incremento de competitividad, proporcionando unos mayores ingresos y reduciendo los costes operacionales. En este sentido, Nguyen et al., (2007) resaltan las ventajas competitivas que los sistemas CRM pueden ofrecer a la empresa: mejoras en la lealtad de clientes, en la calidad del servicio ofrecido y en la optimización de procesos de recogida y difusión del conocimiento de clientes.

Para analizar los resultados del CRM se ha propuesto en la literatura una doble perspectiva (véase Tabla 2): beneficios de tipo financiero (incremento en las ventas o rentabilidad de la empresa) y beneficios de mercado (o de relación con clientes, que se traducen en mejoras en la satisfacción y retención de los mismos). Estos dos tipos de resultados son los componentes principales de lo que hemos considerado en este trabajo como factores de resultado de una implementación exitosa del CRM.
Sin embargo, como se indicó anteriormente, no todo son buenos resultados, sino que también se cometen errores al implementar una estrategia de este tipo, tales como los siguientes (Rigby et al., 2002; Renart, 2004; Finnegan y Currie, 20 I0): poner en marcha una estrategia de CRM sin diseñar antes una estrategia de relación con el cliente; implementar un CRM sin tener suficientemente en cuenta los cambios que deben introducirse en la organización; pensar que cuanta más tecnología, mejor; y asumir que el cliente siempre quiere mantener una relación con la empresa.

En este sentido, uno de los fallos más frecuentes desde el prisma directivo es dar un protagonismo excesivo al aspecto tecnológico. Se tiende a caer en el error de pensar que un proyecto de CRM es, por definición, tecnológicamente intensivo, y que la tecnología es el factor clave en el éxito del mismo. Por el contrario, la realidad demuestra que los objetivos del CRM pueden alcanzarse sin tener que invertir grandes sumas de dinero en tecnología (Renart, 2004).Y cada empresa debe analizar sus necesidades y determinar qué tipo de solución tecnológica (con mayor o menor sofisticación) le aportará un mayor valor a su negocio.

Para analizar los factores claves de éxito en la implementación del CRM hemos analizado diversos estudios empíricos internacionales desarrollados en empresas de servicios (Chen y Ching, 2004; Roh et al.,2005; Chang et al., 2005; Sin et al., 2005; Eid, 2007) y hemos sintetizado los factores considerados más relevantes para el éxito del CRM en los cuatro siguientes: factores organizativos, tecnológicos, de gestión del conocimiento y de orientación al cliente. 
Tabla 2

Beneficios del CRM

\begin{tabular}{|l|l|}
\hline \multicolumn{1}{|c|}{ Beneficios tangibles (financieros) } & \multicolumn{1}{c|}{ Beneficios intangibles } \\
\hline - Incremento de las ventas & - Satisfacción de clientes \\
- Incremento de la rentabilidad & - Retención \\
- Aumento de la cuota de mercado & - Confianza \\
- Reducción de costes internos & - Mejora en el nivel de lealtad de clientes \\
& - Mejora en la calidad del servicio \\
\hline
\end{tabular}

Fuente: Elaboración propia a partir de Chen y Ching (2004), Sin, Tse y Yim (2005)

Los factores organizativos se refieren a aspectos relacionados con la gestión de recursos humanos, liderazgo y estructura organizativa. La puesta en marcha del CRM implica cambios tanto en la forma en que una empresa se organiza, como en sus procesos de negocio (Sin et al., 2005). Por ello, tanto la estrategia como la estructura organizativa y los procesos de negocio deben ser transformados para desarrollar el CRM, ya que el éxito de la misma dependerá de la adecuada sinergia de los sistemas tecnológicos, procesos y personas (Xu y Walton, 2005). En este sentido, el compromiso del personal y, por tanto, aspectos como la formación y la motivación de los empleados y el establecimiento de sistemas de recompensa adecuados, serán determinantes para la puesta en marcha de una estrategia de este tipo.

Respecto a los factores tecnológicos, para implementar una estrategia de CRM es necesario poseer la tecnología adecuada, que permita optimizar los procesos de negocio implicados en la relación con clientes (Chalmeta, 2006; Mendoza et al., 2007). Los sistemas de software CRM permiten a las empresas ofrecer un servicio personalizado, de mayor calidad y a un coste inferior, por lo que, la mayor parte de las actividades que generan una orientación clientecéntrica no serían posibles sin la tecnología adecuada (Sin et al., 2005). No obstante, como afirman Curry y Kkolou (2004), la tecnología CRM no hace referencia solamente a una serie de herramientas y canales de comunicación, sino que supone la integración de estos canales con el resto de la empresa para obtener una visión única del cliente en los diversos puntos de contacto. Por tanto, considerando que la inversión en tecnología CRM debe ser impulsada desde una perspectiva estratégica (Peppard, 2000), asumiremos que el factor tecnológico constituye una condición necesaria pero no suficiente para el éxito del CRM.

Respecto a la gestión del conocimiento, se la puede considerar como el principal subproceso del CRM
(Zablah et al., 2004). Entendemos a la gestión del conocimiento como proceso que implica identificar, adquirir, transformar, difundir, y utilizar el conocimiento relevante para la consecución de los objetivos de la empresa, y para el desarrollo de nuevas oportunidades (Sáiz et al., 20 I0).Por tanto, para llevar a cabo una estrategia CRM de manera efectiva, las empresas deben desarrollar capacidades relacionadas con los procesos de gestión del conocimiento de clientes (Stefanou et al., 2003), capacidades que, al ser difíciles de imitar, se convertirán en fuente de ventaja competitiva (Dans, 200I).

Por último, la orientación al cliente implica situar al mismo como centro indiscutible de todas las actividades de la empresa, con objeto de ir construyendo una relación a largo plazo (Bentum y Stone, 2005). Este factor constituye un bloque fundamental del clima organizativo necesario para el éxito del CRM, y es particularmente relevante para empresas de servicios: una organización fuertemente orientada al cliente será capaz de diseñar mejor sus procesos, ya que la orientación al cliente implica la comprensión adecuada de los mismos con objeto de ofrecerles un mayor valor añadido (Narver y Slater, 1990). Por tanto, debe considerarse que la orientación al cliente y a sus preferencias, supone un prerrequisito indispensable para la implementación exitosa de una estrategia CRM (Bentum y Stone, 2005; Hsieh, 2009).

Estos cuatro grupos de factores de éxito se tomarán como base del estudio empírico que se comenta a continuación.

\section{Estudio empírico en empresas de servicios}

\section{I. Implementación del CRM en empresas españolas}

Como contexto del estudio empírico en empresas de servicios, comentaremos brevemente diversos estu- 
dios empíricos que analizan el grado de implementación y desarrollo de estrategias CRM en España (IMRC, 2005; AECEM-FECEMD, 2007; Penteo, 2008).

En primer lugar, el estudio Prácticas y Tendencias en la Dirección y Gestión de CRM en España, desarrollado por el Information Management Research Center (IMRC, 2005), analizó una muestra de 97 empresas españolas de diversos tamaños y distintos sectores de actividad. Respecto a la visión conceptual del CRM, el estudio mostró que en las empresas encuestadas predominaba un concepto estratégico del CRM: un $74 \%$ consideraban al CRM como estrategia de negocio, mientras que sólo un $18 \%$ lo contemplaban exclusivamente como herramienta tecnológica. Asimismo, los factores considerados como más relevantes por las empresas encuestadas a la hora de implementar un sistema CRM fueron los siguientes: la implicación de la alta dirección, el liderazgo del proyecto, el uso de la tecnología adecuada y la integración de los procesos de negocio.

En segundo lugar, la Asociación Española de Comercio Electrónico y Marketing Relacional (AECEM-FECEMD) desarrolló en el año 2007 el IV estudio de CRM en España. En el mismo, se utilizó una muestra de 200 grandes empresas pertenecientes a diversos sectores de actividad. Como principales resultados del estudio, se observó como las empresas habían incrementado los niveles de inversión en soluciones de CRM respecto a períodos anteriores, siendo conscientes de la importancia de dichas iniciativas de CRM como medio para incrementar su competitividad. Entre los factores de éxito más relevantes para la estrategia CRMdestacaban el involucrar a toda la organización y la orientación al cliente de los procesos de negocio. Con respecto a las dificultades para la correcta implementación del CRM a nivel global, las más mencionadas fueron el no disponer de la tecnología adecuada, la falta de personal cualificado, el coste de la implementación y la falta de apoyo de la alta dirección.

Por otra parte, en un estudio realizado por la consultora Penteo (2008) a empresas de gran tamaño (facturación anual bruta de entre 25 a 75 millones de euros), se concluía con que el mercado de CRM en España no acaba de consolidarse, en contra de las expectativas y las previsiones que año tras año situaban a las soluciones de gestión de clientes como una de las herramientas con mejores resultados. Asimismo, se puso en evidencia cómo un elevado porcentaje de directivos manifestaban haber pasado por experiencias de implementaciones prolongadas y tortuosas, a pesar de contar con herramientas muy potentes desde un punto de vista tecnológico. Dichos problemas estaban resultando un freno a la inversión en dichas iniciativas.

\subsection{Metodología y resultados}

Para analizar los factores de éxito del CRM mencionados, se llevó a cabo un estudio empírico en el sector servicios a nivel nacional, dirigido concretamente a empresas hoteleras españolas, subsector de gran importancia dentro del sector servicios y de gran relevancia económica por su volumen de negocio generado (PWC, 2008). Dicho sector presenta una especial trascendencia dentro de la actividad turística, que viene determinada por su contribución a la generación de empleo estable; por la organización y mentalidad empresarial de sus gestores; así como por su contribución fiscal y generación de rentas (Uriel et al., 200 I). Además, el sector hotelero, al ser un sector intensivo en información, en el que la importancia del servicio al cliente es fundamental, se encuentra inmejorablemente posicionado para aprovechar las ventajas estratégicas que el CRM ofrece (Piccoli et al., 2003). No obstante, a pesar de dicha importancia, y de que a nivel internacional pueden encontrarse distintos estudios que analizan la puesta en marcha del CRM en empresas hoteleras (Luck y Lancaster, 2003;Sigala, 2005; Sigala, 20 I I) en nuestro país sigue existiendo un gap de investigación en la temática. Por dichas razones, el sector hotelero español fue definido como población objetivo del estudio empírico.

Como paso previo al diseño del estudio, realizamos una entrevista exploratoria con profesionales del sector hotelero en la Feria Internacional de Turismo 2007 (feria de turismo más prestigiosa a nivel nacional). En ella observamos que existía un elevado grado de desconocimiento acerca de CRM en el sector, y que los hoteles que estaban implementando dichas estrategias eran principalmente hoteles de categorías superiores.

Respecto a la metodología utilizada, para la recogida de datos se utilizó como herramienta el cuestionario web. Para ello, en 2008 se envió un cuestionario programado y autogestionado, dirigido a directivos de establecimientos hoteleros. La población ascendió a 4.405 hoteles españoles de categorías superiores (3 a 5 estrellas) y se obtuvo una muestra representativa de 3 II hoteles (error muestral del 5,5\%, para un nivel de confianza del 95,5\%).

La información sobre dicha población objetivo fue obtenida de la Guía Oficial de Hoteles España 2007, 
que es editada anualmente por Turespaña (Secretaría de Estado de Turismo y Comercio). La elección de hoteles de categoría superior se debe a dos razones fundamentales. En primer lugar, dichas categorías son las más dinámicas e innovadoras y representan hoteles con una mayor amplitud de características y posibilidades (Claver, Molina y Pereira, 2006). En segundo lugar, los hoteles de categoría superior eran los que mostraban un mayor grado de inversión tecnológica, por lo que pensamos que, a priori, van a mostrar un mayor grado de implementación de iniciativas CRM.

En nuestra investigación se decidió seguir el método de los informantes clave, $y$, se seleccionaron como informantes a los directores de los establecimientos hoteleros. A ellos se les envió el cuestionario y fue- ron ellos quienes proporcionaron la información sobre las variables a analizar. La tasa de respuesta obtenida fue de un 7\%. Dicha tasa, a pesar de no ser muy elevada, fue similar a la obtenida en otros estudios realizados con la misma población objetivo. En este sentido, Claver, Molina y Pereira (2006) realizaron un estudio dirigido a hoteles españoles de 3 a 5 estrellas y obtuvieron un índice de respuesta del $7,7 \%$, muy similar al nuestro.

Sobre los datos obtenidos se analizó es sesgo de no respuesta, comprobando que la muestra se ajustaba correctamente a la población objeto de estudio, es decir, que no presentaba sesgos. Por tanto, los datos obtenidos eran representativos de la población objeto de estudio. Las principales características de las empresas encuestadas aparecen recogidas en la Tabla 3.

Tabla 3

Descripción de los hoteles que conformaban la muestra

\begin{tabular}{|c|c|c|c|}
\hline \multirow[b]{2}{*}{ DIMENSIONES } & \multirow[b]{2}{*}{ VARIABLES } & \multicolumn{2}{|c|}{ MUESTRA } \\
\hline & & $\begin{array}{l}\text { Número } \\
\text { de hoteles }\end{array}$ & (\%) \\
\hline CATEGORÍA & $\begin{array}{l}\text { - } 3 \text { estrellas } \\
\text { - } 4 \text { estrellas } \\
\text { - } 5 \text { estrellas }\end{array}$ & $\begin{array}{r}151 \\
145 \\
15\end{array}$ & $\begin{array}{r}48,6 \\
46,6 \\
4,8\end{array}$ \\
\hline $\begin{array}{l}\text { TAMAÑO } \\
\text { (N. } .^{\circ} \text { CAMAS) }\end{array}$ & $\begin{array}{l}\text { - Familiar (hasta } 100 \text { camas) } \\
\text { - Pequeño (de I0I a I } 50 \text { camas) } \\
\text { - Mediano (de I5 I a } 300 \text { camas) } \\
\text { - Grande (más de } 300 \text { camas) }\end{array}$ & $\begin{array}{r}143 \\
47 \\
68 \\
53\end{array}$ & $\begin{array}{l}46 \\
15,1 \\
21,9 \\
17\end{array}$ \\
\hline $\begin{array}{l}\text { TAMAÑO } \\
\text { (N. }{ }^{\circ} \text { EMPLEADOS) }\end{array}$ & $\begin{array}{l}\text { - Menos de } 10 \\
\text { - Entre } 10 \text { y } 50 \\
\text { - Entre } 50 \text { y } 250 \\
\text { - Más de } 250\end{array}$ & $\begin{array}{c}68 \\
170 \\
66 \\
7\end{array}$ & $\begin{array}{c}21,9 \\
54,7 \\
21,2 \\
2,3\end{array}$ \\
\hline TIPO DE GESTIÓN & $\begin{array}{l}\text { - Independiente } \\
\text { - Pertenece a una agrupación de hoteles independientes } \\
\text { - Pertenece a una cadena explotado en régimen de propiedad } \\
\text { - Pertenece a una cadena explotado en régimen de arrendamiento } \\
\text { - Pertenece a una cadena explotado en régimen de contrato de gestión } \\
\text { - Pertenece a una cadena en régimen de franquicia } \\
\text { - Otro }\end{array}$ & $\begin{array}{l}159 \\
24 \\
81 \\
23 \\
11 \\
4 \\
9\end{array}$ & $\begin{array}{r}51,1 \\
7,7 \\
26 \\
7,4 \\
3,5 \\
1,3 \\
2,9\end{array}$ \\
\hline SEGMENTO & $\begin{array}{l}\text { - Sol y playa } \\
\text { - Urbano } \\
\text { - Rural/naturaleza } \\
\text { - Negocio/congresos } \\
\text { - Golf } \\
\text { - Belleza/Spa } \\
\text { - Temático } \\
\text { - Cultural }\end{array}$ & $\begin{array}{r}107 \\
129 \\
89 \\
80 \\
16 \\
36 \\
9 \\
39\end{array}$ & $\begin{array}{r}21,2 \\
25,5 \\
17,6 \\
15,8 \\
3,2 \\
7,1 \\
1,8 \\
7,7\end{array}$ \\
\hline ANTIGÜEDAD & $\begin{array}{l}\text { - Menos de } 5 \text { años } \\
\text { - Entre } 5 \text { y } 10 \text { años } \\
\text { - Entre } 11 \text { y } 15 \text { años } \\
\text { - Entre } 16 \text { y } 25 \text { años } \\
\text { - Más de } 25 \text { años }\end{array}$ & $\begin{array}{r}100 \\
66 \\
26 \\
44 \\
75\end{array}$ & $\begin{array}{r}32,2 \\
21,2 \\
8,4 \\
14,1 \\
24,1\end{array}$ \\
\hline
\end{tabular}

Fuente: Elaboración propia. 
En cuanto a los resultados obtenidos, respecto al grado de implementación del CRM se observó que un 49,2\% (I 53 hoteles) estaban llevando a cabo una estrategia de este tipo. Para valorar dicho dato, podemos compararlo con los obtenidos en la Encuesta de uso de TIC y Comercio Electrónico en las empresas, desarrollada por el Instituto Nacional de Estadística (INE) anualmente, que incluye la variable disponibilidad de herramientas CRM. En dicho estudio (INE, 2009) se observó que sólo un 24,9\% de las empresas encuestadas a nivel nacional disponían de alguna herramienta CRM para gestionar información de clientes en el período considerado, mientras que un $31,9 \%$ de las empresas de servicios disponían de dichas herramientas (observamos como el sector servicios registraba un grado de utilización del CRM superior a la media global). Por tanto, puede afirmarse que el sector hotelero se encuentra en un estado más avanzado, y superior a la media nacional y a la del sector servicios, en lo que se refiere a implementación de estrategias CRM, elemento que comentamos después en las limitaciones a efectos de interpretación de los resultados.

Centrándonos en los hoteles que afirmaron estar llevando a cabo una iniciativa de CRM, más de la mitad (el 50,3\%) confirmaron que habían transcurrido más de dos años desde su puesta en marcha, por lo que observamos que los hoteles que componen la muestra poseían cierto grado de experiencia y madurez en su utilización. Los establecimientos que aún no estaban implementando el CRM señalaron como principales causas de ello el desconocimiento de soluciones de CRM y las ventajas que ofrecen (39,9\% de los casos). No obstante, un 26,8\% afirmó estar planteando su implementación en un futuro, por lo que puede preverse que los niveles de implementación en el sector se incrementarán de forma notable en próximos periodos.

Asimismo, con objeto de profundizar en los factores determinantes del éxito de una iniciativa CRM y extraer implicaciones a nivel directivo para su implementación exitosa, se realizó un análisis multivariante, utilizando una metodología de ecuaciones estructurales. El análisis de ecuaciones estructurales constituye una poderosa técnica de análisis multivariante cada vez más utilizada en la investigación, que permite examinar simultáneamente una serie de relaciones de dependencia. Una vez analizados los datos comprobamos que no eran normales, por lo que procedimos a buscar una solución alternativa para la estimación de modelos SEM con datos no normales. Para ello, el programa EQS 6.I permite estimar indicadores robustos de bondad del ajuste, así como el estadístico chi-cuadrado robusto, denominado «Satorra-Bentler scaled statistics» (Satorra y Bentler, 200 I). Mediante dicho análisis se contrastó un modelo de éxito, que registraba el impacto de los cuatro grupos de factores mencionados: organizativos, tecnológicos, de gestión del conocimiento y de orientación al cliente, en los resultados del CRM. Todos estos factores fueron medidos utilizando una escala de ítems obtenida de la revisión de la literatura previamente descrita.

Fueron estimados diversos modelos alternativos, con objeto de analizar las relaciones existentes entre las diversas variables, y observar si efectivamente los factores propuestos incidían significativamente en el éxito del CRM, y si estas relaciones eran directas o indirectas. La calidad de ajuste de los distintos modelos obtenidos se realizó desde una triple perspectiva: ajuste global del modelo, ajuste del modelo de medida y del modelo estructural. Se observó que el modelo que mostraba un mejor ajuste era el que incluía a las variables organizativas como antecedente del éxito del CRM en la organización, mediando el impacto del resto de factores en los resultados del CRM (financieros y de mercado).

En este caso, se observó como todos los valores $\mathrm{t}$ calculados (razones críticas) superaban los valores de referencia de 1,96 para un nivel de significación de 0,05 , por lo puede afirmarse que los coeficientes estimados eran estadísticamente significativos (para dichos cálculos se utilizaron estadísticos robustos). El modelo global presentó también unos índices de bondad del ajuste adecuados (RMSEA 0,042/reco-

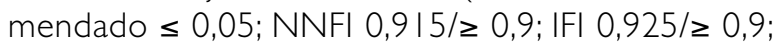
CFI $0,923 / \geq 0,9$ ). Además, su coeficiente de determinación fue de 0,633, por lo que podemos afirmar que un $63,3 \%$ de la variabilidad de los resultados del CRM era explicada por el modelo propuesto.

La estimación estadística del modelo descubrió el papel fundamental que ejercen los factores de tipo organizativo en el éxito de iniciativas CRM. Se observó que, si bien es cierto que los factores tecnológicos, la gestión del conocimiento y la orientación al cliente eran relevantes, los factores organizativos mostraron ser determinantes a la hora de implementar con éxito una estrategia de este tipo. Además, estos factores organizativos (relacionados con el liderazgo y el apoyo de la alta dirección, la estructura organizativa y la gestión del personal) se mostraban como antecedentes del éxito del CRM, ejerciendo un impacto directo, mientras que variables como la tecnología utilizada constituían simplemente una condición necesaria pero no suficiente para el éxito. 
Como consecuencia del estudio empírico realizado, planteamos un mapa de relaciones entre factores que explica cómo realizar una implementación exitosa del CRM (en términos de mercado y financiero) (Véase Figura I).

\section{Implicaciones directivas y consideraciones finales}

Como se ha indicado a lo largo del trabajo, el CRM es un tema relevante para las empresas en la actualidad, ya que aporta numerosas ventajas. Sin embargo, hemos constatado que, a pesar dichas ventajas, la implementación del CRM no está aún consolidada en empresas españolas de servicios.

Hemos descrito los obstáculos a la implementación exitosa del CRM, en términos de mercado y financieros. Por ejemplo, en multitud de ocasiones los directivos otorgan un protagonismo excesivo al ámbito tecnológico. Esto conlleva que no se tengan en cuenta otras cuestiones relevantes como pueden ser la gestión del conocimiento de clientes o el cambio organizativo que el CRM implica, produciendo altos índices de fracaso. Por ello, tras realizar una revisión de la literatura internacional y un estudio empírico en empresas de servicios, podemos proponer un mapa de relaciones de los principales factores determinantes del éxito del CRM. Dicho mapa es de gran utilidad a la hora de poner en marcha una estrategia de este tipo en el sector servicios, ya que identifica los factores organizativos (gestión de recursos humanos, liderazgo y cultura organizacional) como mediadores del resto de factores considerados (gestión del conocimiento, tecnología y orientación al cliente) en el éxito de la implementación del CRM. Además, podemos extraer varias implicaciones directivas.

Como principal implicación directiva podemos mencionar el que el desarrollo e implementación de estrategias CRM en el sector servicios debe estar precedida por un cambio a nivel organizativo. Es decir, la iniciativa sólo tendrá éxito si los procesos de la organización (medidas de resultados, sistema retributivo, programas de formación, etc.) se reestructuran para lograr una mejor comprensión de las necesidades de los clientes. Por tanto, al igual que diversos autores (Rigby et al., 2002; Shum et al., 2008; Chang et al., 20 I 0) hemos constatado que la puesta en marcha de CRM requiere que la empresa adopte una filosofía centrada en el cliente, cambie su estructura y procesos, y modifique su cultura corporativa.

Consecuentemente, podemos enunciar un conjunto de recomendaciones directivas específicas para la implementación exitosa del CRM, es decir, obteniendo resultados positivos tanto a nivel financiero como de

Figura I

Marco conceptual para la implementación del CRM en empresas de servicios

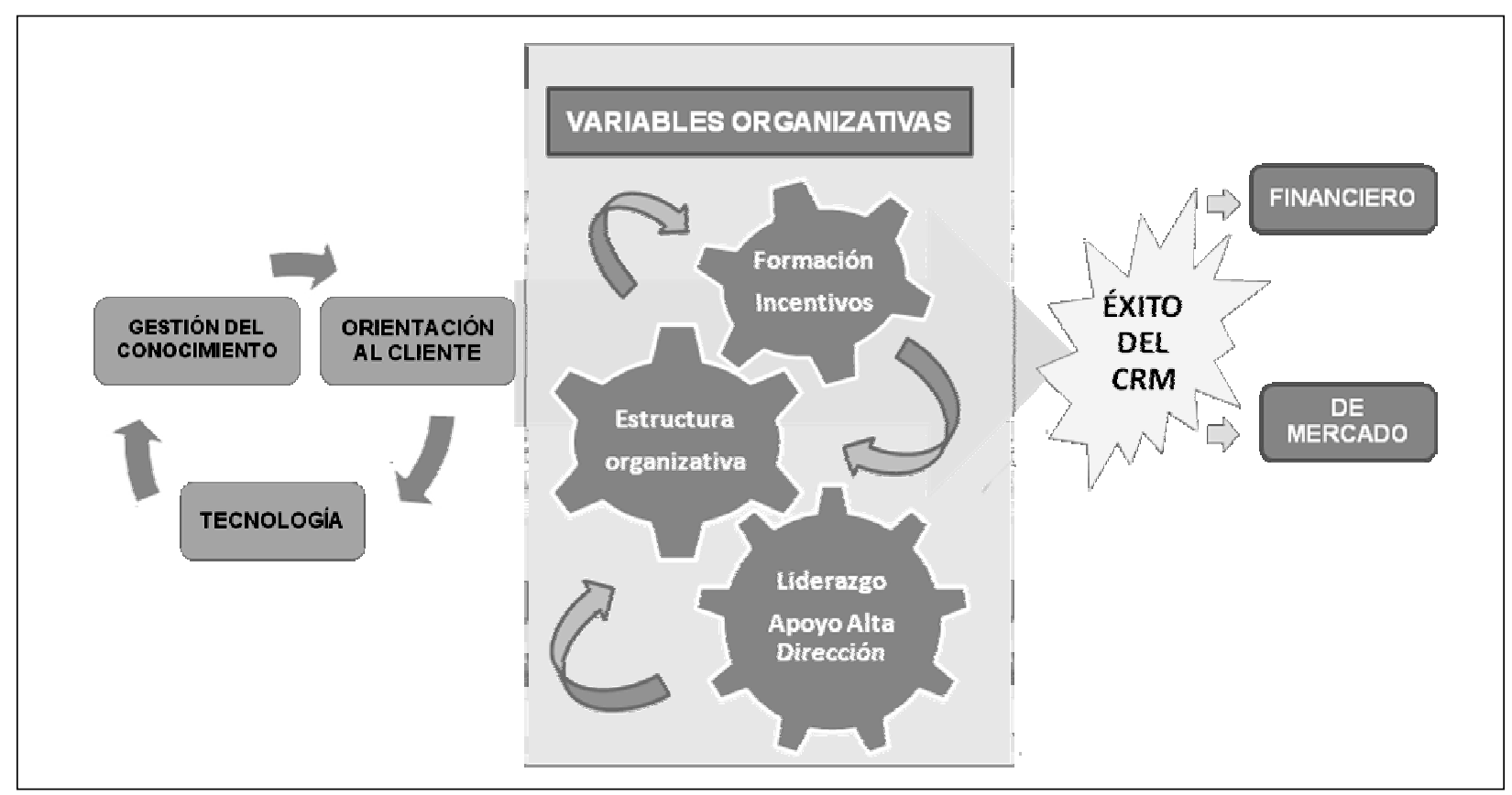

Fuente: Elaboración propia. 
mercado. En primer lugar, los directivos implicados deben llevar a cabo un liderazgo adecuado de la implementación del CRM, haciendo partícipe de los objetivos de la misma al personal de la empresa y mostrando una intensa implicación directiva en su puesta en marcha. En segundo lugar, deben llevarse a cabo una serie de iniciativas relacionadas con la gestión del personal, ya que su compromiso e implicación resultan determinantes para poner en marcha la estrategia. En este sentido, se debe hacer partícipe al personal de los objetivos del CRM, comunicándolos claramente, proporcionando a los empleados la formación necesaria y estableciendo un sistema de retribución y recompensa en base al cumplimiento de dichos objetivos. En tercer lugar, la estructura organizativa debe seguir un enfoque centrado en los clientes, y los distintos departamentos deben trabajar de manera conjunta en la satisfacción de las necesidades de los mismos. Asimismo, los sistemas de información de la empresa deben estar integrados a través de las distintas áreas funcionales, de manera que la información individualizada sobre cada cliente esté disponible en todos los puntos de contacto.

Por tanto, como consideración final se puede decir que, una vez que la empresa cuente con este clima organizativo, una orientación cliente-céntrica, unas capacidades avanzadas de gestión del conocimiento y la tecnología necesaria integrada en las distintas áreas funcionales, la implementación del CRM tendrá los resultados esperados, tanto desde un enfoque financiero como de mercado.

Como limitaciones de este trabajo, cabría mencionar la adelantada ya en el análisis empírico y que se refiere a que las empresas consideradas (hoteles) están por encima de la media del sector servicios en la implementación del CRM lo cual podría limitar la aplicación de los resultados obtenidos y las implicaciones directivas descritas a otros subsectores dentro del sector servicios. Por ello, como futuras líneas de investigación se plantean el contrastar el modelo en otros subsectores del sector servicios como pueden ser: transporte y restauración, servicios financieros u otros servicios empresariales.

\section{Bibliografía}

AECEM-FECEMD (2007). IV estudio de CRM en España. Asociación Española de Comercio Electrónico y Marketing Relacional: Madrid.

AEMR (2002). I estudio del CRM en España. Extraido el 20 de Diciembre de 2005 de: http://www.fecemd.org/archivos/aemr-estudio2002.pdf
BECKER, J. U., GREVE, G., y ALBERS, S. (2009). «The impact of technological and organizational implementation of CRM on customer acquisition, maintenance, and retention». International Journal of Research in Marketing, 26, pp. 207-2I5.

BENTUM, R.V., y STONE, M. (2005). «Customer relationship management and the impact of corporate culture -a European study». Journal of Database Marketing \& Customer Strategy Management, I 3( I), pp. 28-54.

BERRY, L. ( I 983). «Relationship Marketing» in Berry, Shostack, and Upah (eds), Emerging Perspectives on Services Marketing, American Marketing Association: Chicago.

CLAVER, E., MOLINA, y J.F., PEREIRA, J. (2005). «Estrategias y ventajas competitivas de los hoteles españoles y su relación con el desempeño». Papers de Turisme, 37 38, pp. 27-43.

CHALMETA, R. (2006). «Methodology for customer relationship management». The Journal of Systems and Software, 79, pp. I0I5-1024.

CHANG, T.M., LIAO, L.L., y HSIAO, W.F. (2005). «An Empirical Study of the e-CRM Performance Influence Model for Service Sectors in Taiwan». Proceedings of the 2005 IEEE International Conference on e-Technology, e-Commerce and e-Service, pp. 240-245.

CHANG, W., PARK, J.E., y CHAIY, S. (20I0). «How does CRM technology transform into organizational performance? A mediating role of marketing capability». Journal of Business Research, 63, pp. 849-855.

CHEN, I.J., y POPOVICH, K. (2003). «Understanding customer relationship management-people, process and technology». Business Process Management Journal, 9(5), pp. 672-688.

CHEN, J., y CHING, R. (2004). «An Empirical Study of the Relationship of IT Intensity and Organizational Absorptive Capacity on CRM performance». Journal of Global Information Management, I2(I), pp. I- 17.

CHOY, K.L., FAN, K., y LO, V. (2003). «Development of an intelligent customer-supplier relationship management system: the application of case-based reasoning». Industrial Management +Data System, I03(3/4), pp. 263274.

CURRY,A., y KKOLOU, E. (2004). «Evaluating CRM to contribute to TQM improvement -a cross case comparison». The TQM Magazine, I6(5), pp. 314-324.

DANS, E. (200I). «Sobre modas y realidades: CRM o el nuevo marketing digital». Información comercial española. Revista de economía, 791, pp.55-62.

DAS, K. (2009). «Relationship marketing research (19942006). An academic literature review and classification». Marketing Intelligence \& Planning, 27(3), pp. 326-363. 
EID, R. (2007). «Towards a Successful CRM Implementation in Banks: An Integrated Model». The Services Industries Journal, 27(8), pp. I021 - 1039.

FINNEGAN, D.J., y CURRIE,W.R. (2010). «A multi-layered approach to CRM implementation: An integration perspective». European Management Journal, 28, pp. I53167.

GARTNER (2010): Predicts 201 I: CRM Enters a ThreeYear Shake-Up. Extraído el 14 de Junio de 201 I de: http://www.gartner.com/

GRÖNROOS, C. (1994). «Quo Vadis, marketing? Towards a relationship marketing paradigm», Journal of Marketing Management, I0, pp. 347-360.

HSIEH, M.H. (2009). «A case of managing customer relationship management systems: Empirical insights and lessons learned». International Journal of Information Management, 29, pp. 416-419.

IGLESIAS-PRADAS, S.,PASCUAL-MIGUEL, F., CHAPARRO-PELÁEZ, J., y HERNÁNDEZ-GARCÍA, A. (2009). «Influencia de las características del puesto de trabajo en la implantación de TIC móviles en la organización». Dirección y Organización, 38, pp. 47-57.

INFORMATION MANAGEMENT RESEARCH CENTER (2005). «Prácticas y Tendencias en la Dirección y Gestión de CRM en España». Extraído el 16 de octubre de 2010 de http://www.financialtech-mag.com/_docum/37_Marketing_y_Servicios_0I.pdf

INSTITUTO NACIONAL DE ESTADÍSTICA (2009): Encuesta de uso de TIC y Comercio Electrónico (CE) en las empresas 2008-2009. Extraído el 15 de Octubre de 2010 de: http://www.ine.es/

KEVORK, E.K., y VRECHOPOULOS, A. P. (2009). «CRM literature: conceptual and functional insights by keyword analysis». Marketing Intelligence \& Planning, 27( I), pp. 48-85.

LUCK, D., y LANCASTER, G. (2003). «E-CRM: customer relationship marketing in the hotel industry». Managerial Auditing Journal, I8(3), pp. 2| 3-23।.

MENDOZA, L.E,MARIUS, A., PÉREZ, M., y GRIMÁN, A.C. (2007): «Critical success factors for a customer relationship management strategy». Information and Software Technology, 49, pp. 913-945.

NARVER, J.C., y SLATER, F.S. ( 1990). «The Effect of a Market Orientation on Business Profitability». Journal of Marketing, 54(4), pp. 20-35.

NGAI,V.W.T. (2005). «Customer relationship management research (1992-2002). An academic literature review and classification». Marketing Intelligence and Planning, 23(6), pp.582-605.

NGUYEN, T.H., SHERIF, J.S, y NEWBY, M. (2007). «Strategies for successful CRM implementation». Information Management \& Computer Security, I 5(2), pp. I02- I I 5.
PARVATIYAR, A., y SHETH, J.N. (200I). «Customer Relationship Management: Emerging Practice, Process, and Discipline». Journal of Economic and Social Research, 3(2), pp. I-34.

PENTEO (2008). «Las soluciones CRM en España». Extraído el 22 de Octubre de 2010 de: http://www. iese.edu/en/files/Estudios\%20CRM_tcm4-24630.pdf

PEPPARD, J. (2000). «Customer Relationship Management (CRM) in financial services». European Management Journal, I8(3), pp. 312-327.

PRICEWATERHOUSECOOPERS (2008): «La transformación del sector turístico español. Retos y oportunidades». Extraído el 7 de Noviembre de 2010 de: http://www.pwc.com/es_ES/es/sala-prensa/assets/turismo.pdf

REICHHELD, F. F. (1996). The Loyalty Effect:The Hidden Force Behind Growth, Profits and Lasting Value. Harvard Business School Press: Cambridge, MA.

REIMANN, M., SCHILKE, O., yTHOMAS, J.S. (20 I0). «Customer relationship management and firm performance: the mediating role of business strategy»y. Journal of the Academy of Marketing Science, 28, pp. 326-346.

REINARES, P., y PONZOA, J. (2002): Marketing relacional. Un nuevo enfoque para la seducción y fidelización del cliente. Pearson Education: Madrid.

RENART, L.G. (2004). «CRM: tres estrategias de éxito». Cuadernos del e-Business Center PwC \& IESE. Extraído el 8 de Marzo de 2010 de: http://www.iese.edu/en/files/6_13439.pdf.

RIGBY, D., REICHHELD, F, y SCHEFTER, P. (2002). «Avoid the Four Perils of CRM». Harvard Business Review, 80(2), pp. $101-109$.

ROH, T.H, AHN, C.K., y HAN, I. (2005). «The priority factor model for customer relationship management system success». Expert Systems with Applications, 28, pp. 64I-654.

RYALS, L, y PAYNE, A. (200 I). «Customer relationship management in financial services: towards information enabled relationship marketing». Journal of Strategic Marketing, 9, pp.3-27.

SÁIZ, L., MANZANEDO, M.A., DEL OLMO, R., y ALCALDE, R. (20|0). «Propuesta de Modelo de Gestión del Conocimiento para el sector energético. Aplicación empírica». Dirección y Organización, 42, pp. 31-37.

SATORRA, A., y BENTLER, P. M. (200I). «A Scaled Difference Chi-square Test Statistic for Moment Structure Analysis». Psychometrika, 66(4), pp. 507-5 I 4.

SHUM, P., BOVE, L., y AUH, S. (2008). «Employees' affective commitment to change. The key to successful CRM implementation». European Journal of Marketing, 42( I | / 2), pp. |346- | 37|. 
SIGALA, M. (20I I). «eCRM 2.0 applications and trends: The use and perceptions of Greek tourism firms of social networks and intelligence». Computers in Human Behavior, 27, pp. 655-661.

SIGALA, M. (2005). «Customer Relationship Management in Hotel Operations: managerial and operational implications». International Journal of Hospitality Management, 24(3), pp. 391-413.

SIN, L.Y.M, TSE, A.C.B., y YIM, F.H.K. (2005). «CRM conceptualization and scale development». European Journal of Marketing, 39( | | / 2), pp. I 264- 1290.
STEFANOU, C.J., SARMANIOTIS, C., y STAFYLA, A. (2003). «CRM and customer-centric knowledge management: an empirical research». Business Process Management Journal, 9(5), pp. 617-634.

XU, M.; WALTON, J. (2005). «Gaining customer knowledge through analytical CRM». Industrial Management + Data Systems, I05(7), pp. 955-972.

ZABLAH, A.R., BELLENGER, D.N., JOHNSTON, W.J. (2004). «An evaluation of divergent perspectives on customer relationship management:Towards a common understanding of an emerging phenomenon». Industrial Marketing Management, 33, pp. 475-489. 